\title{
Development of synthetic open porous structures for improved heat transfer
}

\author{
Anastasia August ${ }^{\mathrm{a}, \mathrm{c}, *}$, Farshid Jamshidi $^{\mathrm{a}}$, Aron Kneer ${ }^{\mathrm{a}, \mathrm{b}}$, Rainer H. Wolf ${ }^{\mathrm{d}}$, Michael Wirtz ${ }^{\mathrm{b}}$, \\ Britta Nestler ${ }^{\mathrm{a}, \mathrm{c}}$
}

\author{
a Institute of Digital Materials Science, Karlsruhe University of Applied Sciences, Moltkestrasse 30, Karlsruhe 76133, Germany \\ b TinniT Technologies GmbH, Essenweinstr. 25, Karlsruhe 76131, Germany \\ ${ }^{c}$ Institute for Applied Materials, Karlsruhe Institute of Technology (KIT), Strasse am Forum 7, Karlsruhe 76131, Germany \\ d BTE I \&'V GmbH \& Co KG, Weglangstraße 10, Karlsruhe-Hagsfeld 76139, Germany
}

Keywords:

Computational materials design

Heat transfer

Open-pore foams

Tap water heating

\begin{abstract}
A B S T R A C T
If water pipes, which are heated from the outside, are lined with an open porous inner structure, the heat can quickly reach the water via the large contact surface. At the same time, the flow resistance increases due to the presence of the interior. We aim for computationally designing an internal structure that is advantageous for both heat transfer and flow. In this paper we present our path from digital models to metallic pipe components with specifically adjusted properties: First, we design digital models for line pipe elements and qualify them with the help of computer simulations. As a next step, the algorithmically generated samples are printed in 3D. The 3D-printed polylactide models serve as prototypes for the production of metallic pipes, which are then tested in experiments. In this work, three types of inner structures are evaluated: Metal foam, cuboid fins and curved fins. For better comparability, we provide them with almost the same specific surface area and porosity. The use of open-pore metal foams is advantageous for greater heat transfer and fins enable smaller pressure drop.
\end{abstract}

\section{Introduction}

Cellular materials are quite interesting in modern engineering. They are examined for lightweight constructions, for heat and mass transfer processes and for noise reduction. In manufacturing systems those kinds of material seem to have advantages like a huge surface and low weight. They are also machinable, shapeable, coverable and they can be open-pored. Open-pored metallic foams can be produced by the place holder method (casting), galvanically or by sintering. All those three production processes lead to different final products. The place holder method can only be used for foams with a quite high porosity [1], but the advantage is, that the ligaments of the foam are solid afterwards. In the case of the galvanic production, only some materials (exhibiting electrical conductivity) are possible to be processed, and the ligaments of the foam are hollow [2]. Sintering is suitable for production of foams of very low porosity [3].

\footnotetext{
* Corresponding author at: Institute of Digital Materials Science, Karlsruhe University of Applied Sciences, Moltkestrasse 30, 76133 Karlsruhe, Germany.

E-mail addresses: anastasia.august2@kit.edu (A. August), farshid.jamshidi@hskarlsruhe.de (F. Jamshidi), a.kneer@tinnit.de (A. Kneer), wolf.rainer@bte-ing.de (R.H Wolf), m.wirtz@tinnit.de (M. Wirtz), britta.nestler@kit.edu (B. Nestler).
}

Open-pored metal foams are predestined for application as heat exchangers [4-6]. They are permeable for fluids and have a large surface area through which they can exchange heat with the streaming media. Therefore, their flow properties, thermodynamic characteristics and geometric structural features are the subject of numerous experimental and simulative investigations: [7-13]. An overview of the values measured and modelled in recent years for the specific surface area depending on porosity or on PPI number (pores per inch) is given in [14].

The pursuit of the best possible application of these properties motivates the development of modern materials for new efficient energy systems [15]. New materials can be designed on the computer and then printed in 3D before they are actually produced by casting or other processes. Before the production in huge amounts, simulation studies can be carried out in order to validate the quality of the new features and in order to determine the material properties. This way we proceed in this work: We imagine a cellular material that fulfills specific physical requirements like more efficient heat and mass transfer as usual and we find methods to digitally generate a cellular or more generally porous structure fulfilling these physical requirements. Then we transfer the digital model to a simulation model to validate the synthetic design. Afterwards, we establish a 3D print of our digital model which is 


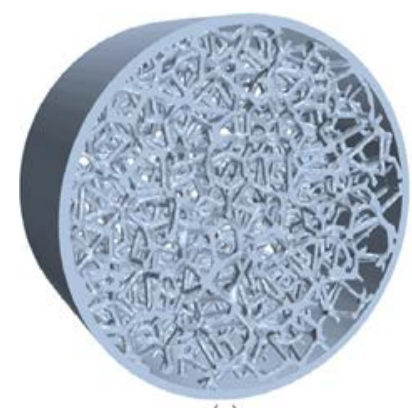

(a)

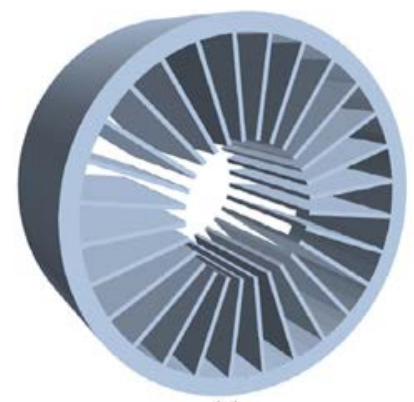

(b)

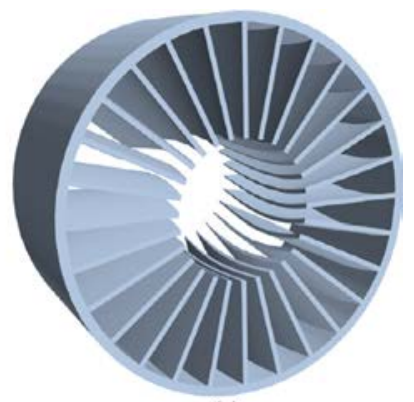

(c)

Fig. 1. Three different designs of a stainless steel pipe element for heating the flowing service water. The specific surface area of these structures as well as the porosity is constructed to be almost identical.

thereafter used as a template to produce a real stainless steel sample. Real experiments are conducted to determine the properties of the sample. The described processing route indicates an innovative way to produce materials with improved and specifically tailored properties - from digital models to metallic samples.

The intended application, which we present in this article, is the heating of tap water. The pipes through which the water flows are heated inductively. If the tubes sheathe a porous structure, the contact surface of the metal to water is higher. The research challenge to be addressed is: Which distribution of the interior equipment increases the heat transfer coefficient particularly well without the pressure loss also increasing excessively?

The lining of the water pipe with open-pored metal foam has proven to be sensible in recent years in research, e.g. [16]. In [17] finned tubes with inner and outer concentric sections are compared with metal-foam filled tube-in-tube heat exchangers.

In this work, we investigate computer models for stainless steel pipes of three various designs, test their properties by means of computer simulations, print PLA (polylactide) samples on a 3D printer, produce stainless steel samples by means of casting, test them in experiments and finally give a comparison of the relevant parameters for the models involved.

\section{Digital material design}

\subsection{Digital models}

The shape of a foam element integrated into a water pipe influences the physical behavior of both the flow and the heat transport. To reduce the pressure loss of the water flow, we may generate foam structures with high porosity and thus with a low inner surface area which directly affects the inner friction. If we imagine that a cellular structure is immersed in a current, then we always have to deal with a flow along the inner wall and a flow around ligaments. The pressure loss resulting from this flow situation is a combination of pressure losses of these two flow phenomena. On the other hand the exchange surface area should be large for a better heat transfer. So we have to consider the design of porous structures in such a way that the inner surface and the number and diameter of the foam ligaments are optimized. And are there probably better arrangements of inner equipment of the tube than the foam structure? Fig. 1 shows besides a synthetic meal foam also two selected fin geometries (which are denoted as fins or wings hereafter) which are analyzed in this work. It can be speculated that the flow through the finned structures is substantially different from the flow through open-pored foam structures. A tube with inner fins builds sub-channels, and each of it is flown through by a portion of the volume rate. So the flow is directed by the fins because of their guiding principle. If the fins are curved (Fig. 1(c)), swirls can be produced to enhance turbulence and heat transfer. How do these geometries compare with heterogeneous open pored structures concerning pressure loss and heat transfer?

\subsection{Design criteria}

The pressure loss of a flow in a pipe depends on the flow behavior (laminar or turbulent), the wall roughness $f$, the fluid properties like the density $\rho$, the dynamic viscosity $\mu$ and the average fluid velocity $u$. However, the standard equation for pipes is well known and is given as

$\Delta p=f \cdot \frac{L}{D} \cdot \frac{\rho}{2} \cdot u^{2}$

whereas $L$ is the length of the pipe, $D$ the diameter of the pipe. For turbulent flow in hydraulically smooth pipes and for a Reynold's Number $R e=\frac{u \cdot D \cdot \rho}{\mu}$ below $10^{5}$, the friction number can be calculated by the Blasius-equation

$f=\frac{0.316}{R e^{0.25}}$.

If a laminar flow regime is considered, the friction number can easily be calculated by $f=64 / R e$. So the pressure loss is well described for pipes without any foam/fin inlet. In case of embedded metal foams and fin structures, these correlations can not be used. For metal foams (Fig. 1(a)), the following Darcy-Forchheimer correlation has been identified to be useful

$\Delta p=L \cdot\left(\frac{\mu}{K} \cdot u+\frac{c_{f} \cdot \rho}{K^{1 / 2}} \cdot u^{2}\right)$.

For a laminar flow regime, the quadratic term on the right side of the equation is very small so only the left term which is called the Darcy-term contributes to the pressure loss. The permeability $K$ and the inertia coefficient $c_{f}$ are unknowns and need to be evaluated by experiments or by numerical analysis. The velocity $u$ is the Darcy velocity and the superficial velocity can be expressed by

$U=\frac{u}{\Psi}$

whereas $\Psi$ represents the porosity of the porous region. Another correlation to calculate the pressure loss $\frac{\Delta p}{\Delta L}$ of the distance $\Delta L$ uses the dimensionless Hagen Number $H g=\frac{\Delta p}{\Delta L} \frac{d_{\mathrm{H}}^{3}}{\rho v^{2}}$. Here $v$ notes the kinematic viscosity of the fluid and $d_{\mathrm{H}}$ the hydraulic diameter of the solid system (see Table 2).

The correlation of the pressure loss for open-cell foams is presented in [14, Eq. (4)] which uses the Hagen-number defined as

$H g=110 \operatorname{Re}_{\Psi}+1.45 \operatorname{Re}_{\Psi}^{2}$.

Here $R e_{\Psi}=\frac{u d_{\mathrm{H}}}{\Psi_{\nu}}$ is the porosity Reynold's number. To evaluate the simulated flow field, the following equation for the pressure 
loss is used

$\Delta p=\frac{\rho v^{2} \Delta L}{d_{\mathrm{H}}^{3}}\left[110 \frac{d_{\mathrm{H}}}{D} \frac{R e}{\Psi}+1.45\left(\frac{d_{\mathrm{H}}}{D} \frac{R e}{\Psi}\right)^{2}\right]$.

In the case of fin and wing configurations (Fig. 1(b) and 1(c)), we can approximately calculate the pressure drop for fin (resp. wing) pipes by an equilibrium consideration as will be discussed in Section 3.2.3.

The Nusselt number $\mathrm{Nu}$ is used to describe the convective heat transfer compared to the pure heat conduction in the system solid structure and flowing liquid (or gas)

$N u=\frac{\alpha L_{C}}{\lambda_{\mathrm{F}}}$.

Here $\lambda_{F}$ is the thermal conductivity of the fluid, $L_{C}$ is a characteristic length of the gestured structure, i.e. the diameter of the pipe $D$ passed through, and $\alpha$ is the heat transfer coefficient. $\alpha$ defines the proportionality factor between the specific power provided $\dot{q}$ and the temperature increase achieved $\Delta T=T_{\mathrm{W}}-T_{\mathrm{F}}$, where $T_{\mathrm{W}}$ is the wall temperature and $T_{\mathrm{F}}$ is the fluid temperature

$\dot{q}=\alpha \Delta T$

For the determination of $\alpha$ we use the enthalpy balance: The power $\dot{Q}$ is the product of the mass flow $\dot{m}$ through the structure, the heat capacity $c_{p}$ of the fluid and the achieved temperature increase after flowing through the pipe

$\dot{Q}=\dot{m} c_{p}\left(T_{\mathrm{O}}-T_{\mathrm{I}}\right)$,

where $T_{\mathrm{I}}$ is the inlet temperature and $T_{\mathrm{O}}$ is the outlet temperature. On the other hand, it results from Eq. (8)

$\dot{Q}=\alpha A_{\mathrm{W}}\left(T_{\mathrm{W}}-T_{\mathrm{F}}\right)$,

where $T_{\mathrm{W}}$ is the wall temperature and $T_{\mathrm{F}}$ is the fluid temperature. This results in $\alpha$ by means of

$\alpha=\frac{\dot{m} c_{p}\left(T_{\mathrm{O}}-T_{\mathrm{I}}\right)}{A_{\mathrm{W}}\left(T_{\mathrm{W}}-T_{\mathrm{F}}\right)}$

If $\dot{Q}$ can completely be transferred into the fluid, the outlet temperature can be calculated based on the energy conservation using Eq. (9). In this case, no heat losses are considered. It is much more interesting to know about the heat transfer coefficient $\alpha$ of foam as well as of fin structures. Resolving these structures within a numerical model and applying the heat flux (given by the inductive heating) as boundary condition make possible to calculate the heat transfer coefficient from Eq. (11). By doing so, the temperature distribution in the resolved structure can also be determined. It is obvious that a small heat transfer coefficient leads to a higher temperature in the structure to compensate for the low heat transfer, and conversely, high heat transfer coefficients reduce the temperature in the structure. The numerical model described in the following sections is used to solve the fully coupled energy equations for fluid and structure as a "numerical experiment". The averaged fluid and structure temperatures and the temperature difference between inlet and outlet are extracted from the numerical simulations and used to calculate $\alpha$ from Eq. (11).

\subsection{Foam characteristics}

An example of a pipe filled with an open foam structure is shown in Fig. 1(a). The structure is synthetically generated applying a specifically designed filling algorithm described in [13]. The fairly high thermal conductivity of metal compared to the thermal conductivity of fluids makes it reasonable to use particularly open fine-pored foams in order to distribute metal as homogeneously as possible inside the pipe and to constitute as large specific surface as possible. This means that the flowing water is not only able to take the heat from the pipe shell area but is additionally warmed at a large number of additional heat sources inside the pipe. On the other hand, too many ligaments imply many obstacles for the streaming water, which induce a large pressure loss. Larger pores and fewer ligaments are presumably more favourable to establish low pressure loss.

An open-pored foam structure - whether made of metal or another solid material - is initially characterized by the following geometric parameters.

The ligaments forming the grid-like network have a certain thickness. This thickness is only subject to a few fluctuations within a homogeneous foam sample. The ligaments meet in the so-called knots. The voids of the foam form the pore space, which is composed of the individual pores. Each pore has a radius, and these radii vary only slightly within a homogeneous sample. The pore space is generally continuous.

The volumetric proportion of the void space to the sum of the void space and the space of the ligaments is called the porosity of the foam.

The choice of solid determines the thermal, electrical and largely mechanical properties of the foam samples.

\subsection{Synthetic foam generation}

The algorithm described in detail in [13] generates open-pored structures with the help of a modified Voronoi decomposition. The input parameters are the domain size, the ligament thickness, the pore radius and the cross-section shape of the ligaments (round or triangular). An average value and a variance in terms of Gaussian distribution can be specified for the ligament thickness and for the pore radius. Special tools can be used to determine the specific surface area and porosity of the generated structure. The specific surface area is measured by counting the surface cells in the surface grid weighted with their surface area. The result is then divided by the volume oft he whole system. To determine porosity, the volume cells of the inner solid structure are measured weighted with their volume content and then divided by the volume of the whole system. The result is subtracted from the one.

\subsection{Other open porous structures}

Open-pored metal foams seem to be particularly suitable for heat exchange with the fluid flowing through them. However, since they can cause a large pressure drop and thus high operating costs, we consider other structures in comparison.

A pretty conceivable structure is the finned tube, Fig. 1(b). The fins also have a large surface area through which heat can be transferred to the flow medium. Due to their orientation along the flow direction, however, they only form an insignificant obstacle to the flow, so that the pressure loss is supposably low.

In order to achieve a better mixing of the water in comparison to the finned tubes, we investigate the third structure, Fig. 1(c). It is similar to the fin structure, but the single fins are slightly curved and their thickness is tapered in the direction of flow, imitating a wing shape. Its profile is from the Eiffel 10 (Wright) designed by Wright brothers.

Pipes are designed and analysed with three types of permeable structures: a) open-pore metal foam, b) a fin structure, c) a wing structure, (Fig. 1). All three structures have approximately the same specific surface area and largely the similar porosity.

Being made of stainless steel such pipes can be used to heat household water. The heat amount can be applied by heating the pipe sheath and diffuse inside of the porous metal structure. Due to the large metal-water interface, the heat can be efficiently released into the water. The research challenge is, to identify the 


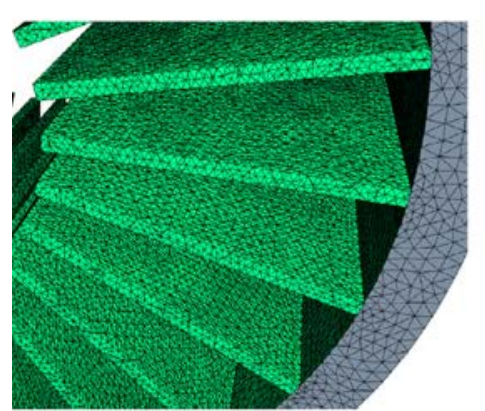

(a)

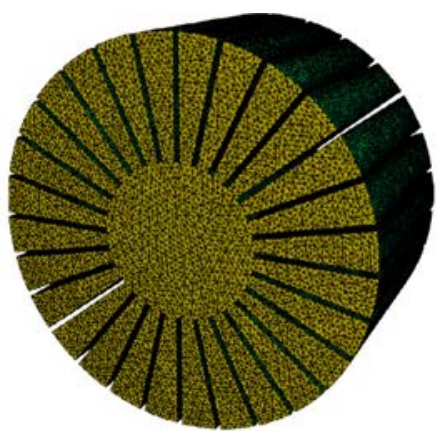

(b)

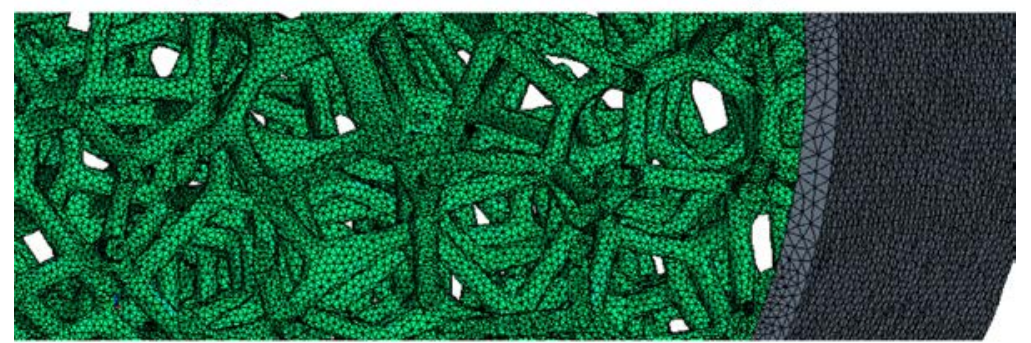

(c)

Fig. 2. (a): The surface mesh of the fins. (b): The surface mesh of fluid region. (c) The surface mesh of the foam ligaments.

optimal structure that fulfills the combined task of efficient heat transport and low pressure loss.

\section{From digital to numerical model}

\subsection{Numerical model generation}

A numerical simulation mesh is engendered from a digital model that resolves the geometry of the system sufficiently well without the number of volume cells being too large and thus too computationally intensive. In the commercial CFD solver STAR$\mathrm{CCM}+$, a suitable surface grid is generated. Fig. 2(a) shows the surface network of the outer side of the tube and of the fins. Since the heat diffusion in the stainless steel structure is to be simulated predominantly radially, the number of surface cells to be seen in the radial direction is significant.

Fig. 2 (b) shows the surface grid of the inner fluid filled region of the finned tube. This region is to simulate the transport of mass and heat by means of flowing water. The large number of surface cells is necessary to enable the formation of boundary layers. Fig. 2 (c) shows a section of the grid of the foam structure in the pipe.

Once the surface grid is compiled, the matching volume grid is to be produced. To make the volume network visible, cuts are usually made through the regions. Fig. 3 shows a section along the tube axis that gives a sense of the amount of volume cells necessary to model the mass and heat transport in the tube physically correctly. In particular, a forward flow and a long last running flow of the fluid region are required. We have chosen a forward flow with the length of the pipe and a last running flow with the length five times longer than the test pipe. These regions may be resolved somewhat coarser because they do not contain complex geometry.

Overall, the simulation area has more than 3.6 million volume cells (3 612924 cells).

\subsection{Numerical experiments for flow and heat transfer analysis}

We performed several heat transport and flow simulations.

\subsubsection{Validation}

The simulations are validated by respective experiments described in Section 4 allowing a comparison of the temperature increase of water streaming through a pipe filled with open-cell metal foam, shown for example in Fig. 13(c). The pipe is heated inductively from outside. To reduce computing time of the simulations, one seventh of this pipe in considered by setting the power entered per square meter and the inflow velocities as boundary conditions recovering the experimental setup. Simulation studies are performed varying the inflow velocities and heat inputs.

The simulation parameters are summarized in Table 1 .

The temperature difference between the outflowing and inflowing water was evaluated. The results are presented in Fig. 4. A higher energy input causes a stronger temperature increase, which becomes smaller with increasing inflow velocity.

Fig. 4 shows a comparison between the simulated and the experimental temperature increase of a pipe with inner open-cell metal foam produced by Selective Laser Melting (SLM) illustrated in Fig. 13(c). Quantitatively, a good agreement is found between the simulative and the experimental values for the temperature increase. However, it is noticeable that the difference between the simulations and the experiments increases with the increasing applied induction power. This is a sign that above a certain value it is not possible to bring all the available power into the system. Simulations, on the other hand, do not take the losses into account and predict a higher temperature rise than is actually measured in the experiment.

\subsubsection{Three different designs}

In order to compare different geometries with each other, we have performed simulations to investigate heat transport and flow properties. Water flow is simulated through externally heated stainless steel pipes with open-pored metal foam, a fin structure or a wing structure. Fig. 1 displays the considered pipe elements. The heating is modelled as a constant boundary condition (heat flow $\dot{Q}$ through the outer sheath). Water of temperature $8^{\circ} \mathrm{C}$ flows in at a constant speed $u$ between $0.01 \frac{\mathrm{m}}{\mathrm{s}}$ and $0.34 \frac{\mathrm{m}}{\mathrm{s}}$ on one side. The heat induced via the outer shell flows into the inner structure and is released into the water via the contact surface. The water trans- 


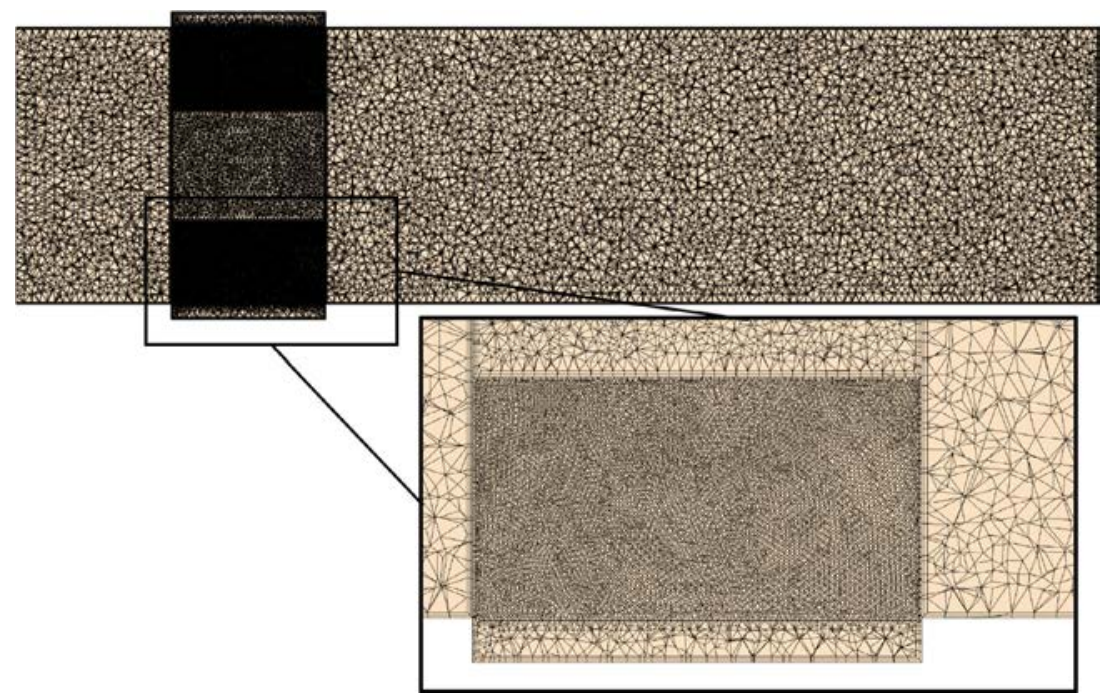

Fig. 3. Cross section through the domain: The volume mesh.

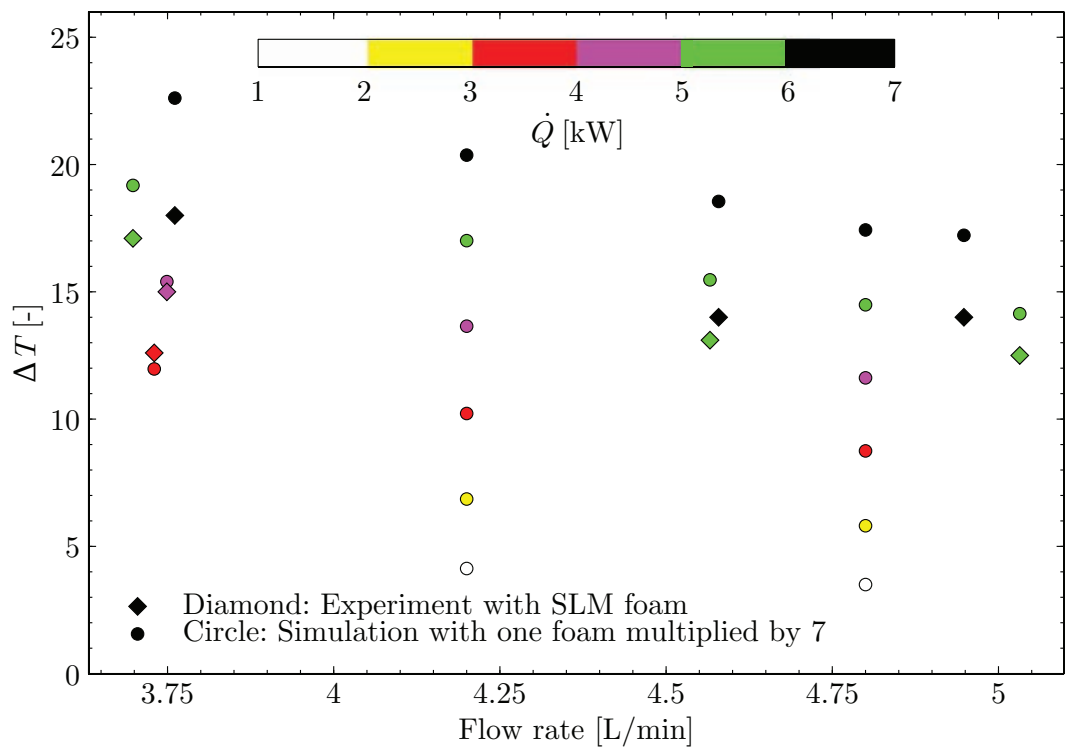

Fig. 4. Comparison between simulations and experimental results.

Table 1

Simulation parameters for the validation of the solver by means of the experiments.

\begin{tabular}{lll}
\hline & & foam pipe, Fig. 1(a) \\
\hline$L$ & pipe length & $20.1 \mathrm{~mm}$ \\
$D$ & inner diameter & $38 \mathrm{~mm}$ \\
& outer diameter & $40 \mathrm{~mm}$ \\
$A$ & surface area & $0.0149 \mathrm{~m}^{2}$ \\
$\Psi$ & porosity in the inner space & 0.861 \\
$\dot{q}$ & heat flux through the outer pipe surface & $69645 \frac{\mathrm{W}}{\mathrm{m}^{2}} \ldots 348227 \frac{\mathrm{W}}{\mathrm{m}^{2}}$ according to $\dot{Q}=1200 \mathrm{~W} \ldots 6000 \mathrm{~W}$ \\
$T_{\mathrm{I}}$ & inflow water temperature & $8.0^{\circ} \mathrm{C} \ldots 18.7^{\circ} \mathrm{C}$ \\
$u$ & inflow velocity & $0.061 \frac{\mathrm{m}}{\mathrm{s}} \ldots 0.082 \frac{\mathrm{m}}{\mathrm{s}}$ \\
$S_{V}=\frac{A}{\frac{\pi}{4} D^{2} L}$ & volume-specific surface area & $653.63 \frac{\mathrm{m}^{2}}{\mathrm{~m}^{3}}$ \\
\hline
\end{tabular}

ports the heat: diffusely and advectively, and continuously warms up while passing through the porous structure.

The selected simulation parameters are summarized in Table 2 . The specific surface of all structures is kept approximately the same, as well as the porosity. Selected constant speeds $(u)$ are $0.01,0.02,0.05,0.07,0.12,0.21,0.28$ and $0.34 \frac{\mathrm{m}}{\mathrm{s}}$, corresponding to Reynolds numbers $\left(R e=\frac{u L_{C}}{v}=\frac{u D}{v}\right.$ with $\left.v=8.91 \cdot 10^{-7} \frac{m^{2}}{\mathrm{~s}}\right)$ of approx. 500, 1000, 2000, 3000,6000, 9000, 12000 and 15000 .

3.2.3. Pressure drop in the fin structure

The fluid flow through pipes with fin configuration (Fig. 1(b)) can be split into two types: a flow through the inner circular 
Table 2

Simulation parameters for simulation studies.

\begin{tabular}{lllll}
\hline & & foam pipe & fin pipe & wing pipe \\
\hline$L$ & pipe length & $20.1 \mathrm{~mm}$ & $20.1 \mathrm{~mm}$ & $20.1 \mathrm{~mm}$ \\
$D$ & inner diameter & $38 \mathrm{~mm}$ & $36 \mathrm{~mm}$ & $36 \mathrm{~mm}$ \\
& outer diameter & $40 \mathrm{~mm}$ & $40 \mathrm{~mm}$ & $38 \mathrm{~mm}$ \\
$A$ & surface area & $0.0149 \mathrm{~m}^{2}$ & $0.0147 \mathrm{~m}^{2}$ & $0.0149 \mathrm{~m}^{2}$ \\
$\Psi$ & porosity in the inner space & 0.861 & 0.846 & 0.897 \\
& number of fins or wings & & $28 \mathrm{fins}$ & 28 wings \\
$\dot{q}$ & heat flux through the outer pipe surface & $646000 \frac{\mathrm{W}}{\mathrm{m}^{2}}$ & $646000 \frac{\mathrm{W}}{\mathrm{m}^{2}}$ & $646000 \frac{\mathrm{W}}{\mathrm{m}^{2}}$ \\
$T_{\mathrm{I}}$ & inflow water temperature & $8 \circ \mathrm{C}$ & $8^{\circ} \mathrm{C}$ & $\mathrm{C}$ \\
$S_{V}=\frac{A}{\frac{\pi}{4} D^{2} L}$ & volume-specific surface area & $653.63 \frac{\mathrm{m}^{2}}{\mathrm{~m}^{3}}$ & $718.50 \frac{\mathrm{m}^{2}}{\mathrm{~m}^{3}}$ & $728.27 \frac{\mathrm{m}^{2}}{\mathrm{~m}^{3}}$ \\
$d_{\mathrm{H}}=4 \frac{\Psi}{S_{V}}$ & hydraulic diameter & $5.27 \mathrm{~mm}$ & $4.71 \mathrm{~mm}$ & $4.93 \mathrm{~mm}$ \\
\hline
\end{tabular}

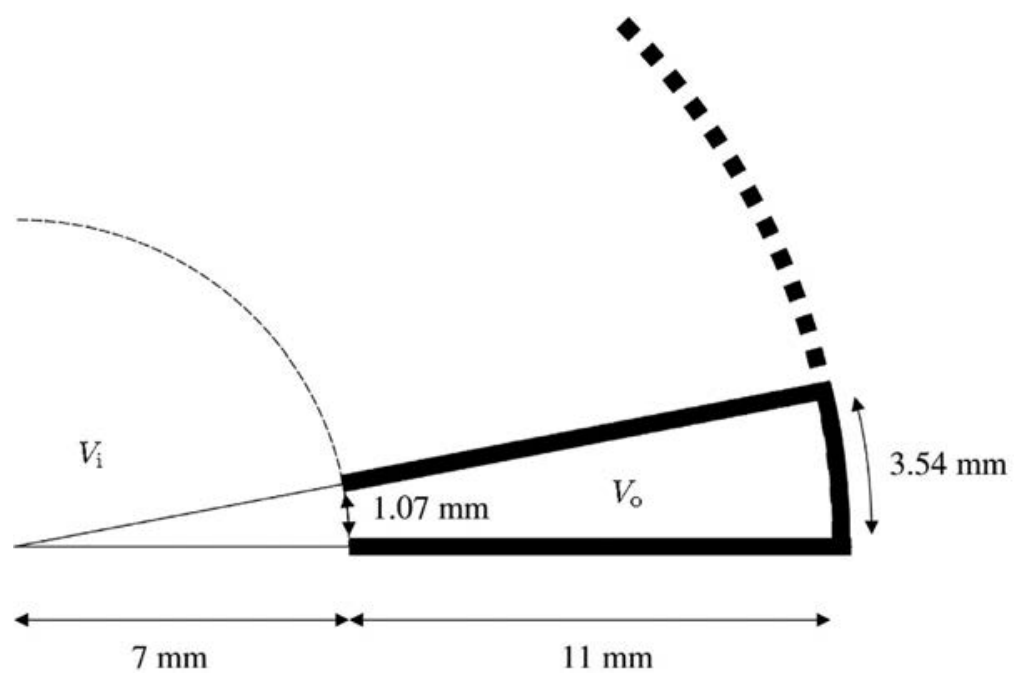

Fig. 5. Segment of outer volume.

space (without fins), and a flow through one of 28 segments, composed of two neighbor fins and an outer wall arc. We calculate the pressure drop for each type. In the equilibrium state, the total volume flow will split in such a manner, that the pressure drop for both types will be the same because otherwise, a volume flow exchange between the types could lower the overall pressure drop.

By design, there is an empty inner circular volume $V_{\mathrm{i}}$ of diameter $d_{\mathrm{i}}=14 \mathrm{~mm}$, and 28 fins, each of width $0.5 \mathrm{~mm}$ and of radial length $11 \mathrm{~mm}$. Two neighbor fins, together with a part of the outer wall, form an outer flow volume $V_{\mathrm{o}}$ with approximate side lengths $1.07 \mathrm{~mm}, 11 \mathrm{~mm}, 3.54 \mathrm{~mm}$ and $11 \mathrm{~mm}$ as illustrated in Fig. 5 . Using the hydraulic diameter $d_{\mathrm{H}}=4 A_{\mathrm{o}} / U_{\mathrm{o}}=3.97 \mathrm{~mm}$, where $A_{\mathrm{o}}$ is the flown-through cross-section and $U_{0}$ the involved circumference, we can calculate the pressure loss for turbulent flow for the outer flow volume. For laminar flow, we suppose the cross-section to be nearly rectangular. Then we can use a correction factor $\varphi$ for the friction number $f=\varphi \frac{64}{R e}$, depending on the aspect ratio of the rectangles sides [18] (p. 1227, Fig. 6). With an aspect ratio of 0.21 we get $\varphi=1.19$.

The inner volume and the outer volumes are not separated by walls. Nevertheless, we may assume friction, as the flow velocities for the two volumes differ by a factor of about 14.8 resp. 2.4 for $R e=500$ resp. $R e=15000$, as we will see. Moreover, the inner channel is delimited by the fin ends, contributing about one-third of the area of an assumed wall.

The overall volume flow $\dot{V}$ will split as

$\dot{V}=\dot{V}_{\mathrm{i}}+28 \cdot \dot{V}_{\mathrm{o}}$,

$\Delta p_{\mathrm{i}}=\Delta p_{\mathrm{o}}$ where $\dot{V}_{\mathrm{i}}$ is the flow in the inner volume, $\dot{V}_{\mathrm{o}}$ is the flow in each of the 28 outer volumes and $\Delta p_{\mathrm{i}}$ and $\Delta p_{\mathrm{o}}$ are the corresponding pressure losses. The equations can be solved iteratively.

If we denote by $x_{\mathrm{i}}=\dot{V}_{\mathrm{i}} / \dot{V}$ the fraction of the inner volume flow, and assume a constant temperature of $T=8^{\circ} \mathrm{C}$, we get the values shown in Table 3.

The asymptotic values of $x_{\mathrm{i}}$ for the considered fin pipe for very small resp. very large velocities $u$ are 0.7625 resp. 0.3420 . These values depend on the temperature $T$.

\subsection{Results discussion and structure qualification}

Figs. 6 and 7 show the temperature distribution in the system in the stationary state. The computed temperature distribution shows that the inner structure of the foam or the fin (wing) structure is not heated up significantly. This means that most of the heat transfer to the fluid occurs already near the inner wall of the tube. Fig. 7 shows the computed temperature distribution in the plane through the fluid part.

The heat penetration into the fluid develops better for the foam and the wing part than for the fin part. The thermal boundary layer can be increased using the foam or the wing structure. There are different reasons for that. First of all, we have to consider the Biot-number which describes the ratio between convection and conduction whereas $\lambda_{S}$ denotes the thermal conductivity of the structure and $\alpha$ the convective heat transfer coefficient reading

$B i=\frac{\alpha L_{C}}{\lambda_{S}}$

The characteristic length $L_{C}$ of the geometry considered describes the ratio between the body volume $V$ and the heated or 


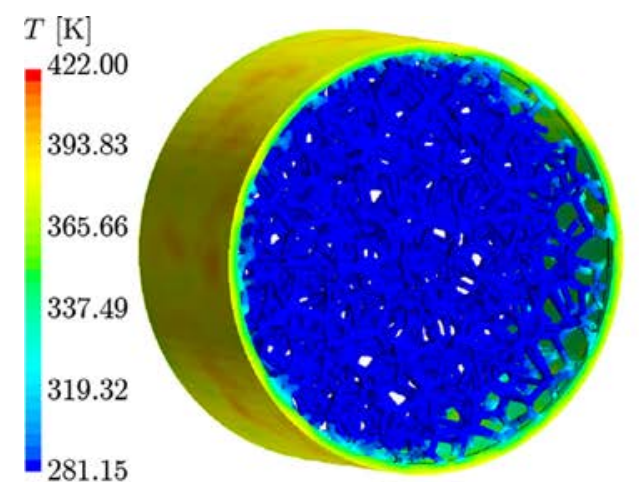

(a)

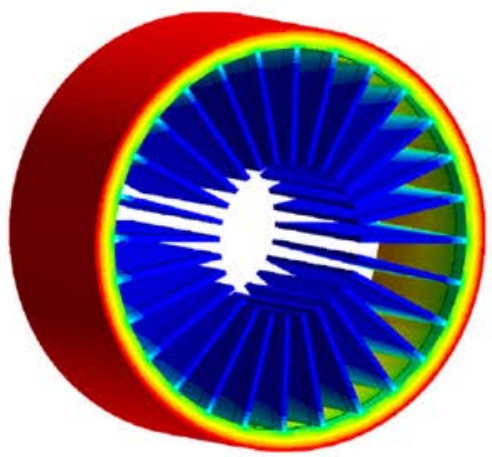

(b)

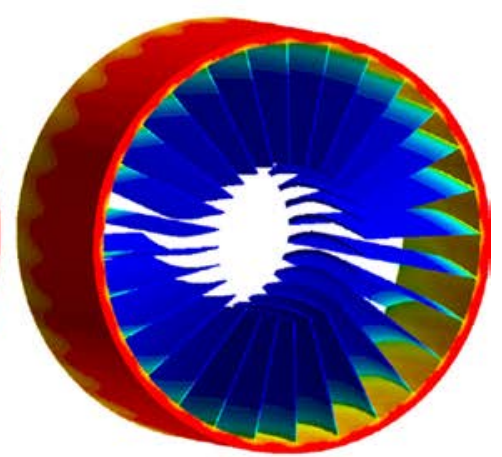

(c)

Fig. 6. Temperature distribution in the solid structure in steady-state for Reynolds number of 15,000 .

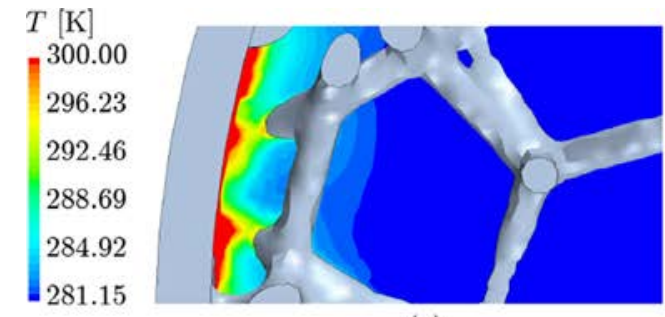

(a)

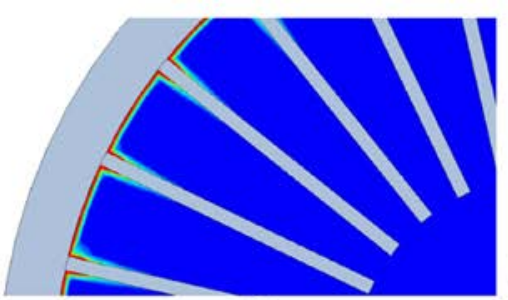

(b)

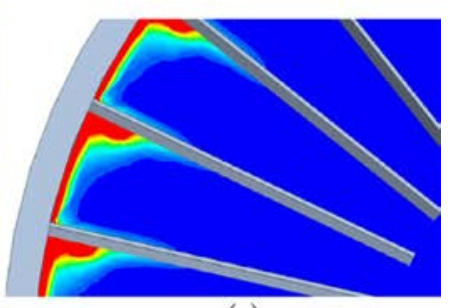

(c)

Fig. 7. Temperature distribution in the fluid in steady-state for Reynolds number of 15,000 .

Table 3

Pressure loss for the fin pipe.

\begin{tabular}{llllllll}
\hline$R e$ & $\dot{V}\left[\mathrm{~m}^{3} / \mathrm{s}\right]$ & $x_{\mathrm{i}}$ & $R e_{\mathrm{i}}$ & $u_{\mathrm{i}}[\mathrm{m} / \mathrm{s}]$ & $R e_{\mathrm{o}}$ & $u_{\mathrm{o}}[\mathrm{m} / \mathrm{s}]$ & $\Delta p[\mathrm{~Pa}]$ \\
\hline 500 & $1.259 \cdot 10^{-5}$ & 0.7625 & 980.3 & 0.0624 & 18.78 & 0.00421 & 0.182 \\
1000 & $2.519 \cdot 10^{-5}$ & 0.7625 & 1961 & 0.125 & 37.6 & 0.00843 & 0.364 \\
2000 & $5.038 \cdot 10^{-5}$ & 0.6025 & 3099 & 0.197 & 126 & 0.0282 & 1.22 \\
3000 & $7.556 \cdot 10^{-5}$ & 0.5484 & 4231 & 0.269 & 214 & 0.0481 & 2.08 \\
6000 & $1.511 \cdot 10^{-4}$ & 0.4558 & 7032 & 0.447 & 516 & 0.116 & 5.00 \\
9000 & $2.267 \cdot 10^{-4}$ & 0.4036 & 9341 & 0.594 & 849 & 0.190 & 8.22 \\
12000 & $3.023 \cdot 10^{-4}$ & 0.3683 & 11360 & 0.723 & 1199 & 0.269 & 11.6 \\
15000 & $3.778 \cdot 10^{-4}$ & 0.3420 & 13190 & 0.839 & 1561 & 0.350 & 15.1 \\
\hline
\end{tabular}

cooled surface $A_{Q}$ of the body expressed as

$L_{\mathrm{C}}=\frac{V}{A_{Q}}$.

In the case of our metal foam, the inner structure has a volume of about $V=3.16 \cdot 10^{-6} \mathrm{~m}^{3}$ and the inner surface is about $A_{\mathrm{i}}=0.0149 \mathrm{~m}^{2}$. We consider that heat transfer occurs only at $A_{Q}$ which is lower than $A_{\mathrm{i}}$ (the inner heat exchanging surface of the foam $A_{f}+$ inner wall $A_{w}$ of the tube). If we assume that $10 \%$ of the foam surface $A_{f}$ participates in heat transfer the respective surface can be calculated by $A_{Q}=0.1 \cdot A_{f}+A_{w}$. With this values in our simulations the Biot-number is between 0.157 and 0.686 , therefore greater than 0.1 . So convection predominates and the incoming energy is therefore very quickly carried away by the flow. As a result, the conductive structures of the ligaments are not really effective, in accordance to the observation in the simulations.

Additionally, the Prandtl-number $\left(\operatorname{Pr}=\frac{v}{a}\right)$ which is linking the velocity field with the temperature field of a fluid is for water about 7 at a temperature of $20 \hat{A}^{\circ}$ C. It represents a measure of the development of the thermal boundary layer (ratio between the flow boundary layer and the thermal boundary layer). Both physical aspects described by $B i$ and $P r$ have to be considered to understand how heat transfer to the fluid can be improved using cellu- lar structures like metal foams or other structures like fins. Unfortunately there exists no empirical correlation for the temperature boundary development of the wing or fin structures (see [19]). Insights of the properties can only be gained numerically.

Nevertheless, regarding the results from the numerical analysis (see Figs. 6 and 7) all structures in the fluid only have a limited contribution to the overall heat transfer to the fluid. Since the induction heating allows only small penetration depths, the heat transfer occurs from the outer wall (heat flux boundary condition) to the inner wall of the pipe.

In Section 3.2.3 we analyzed the pressure drop arising in the fin structure. The results of the iterative solution of the Eqs. (12) and (13) are shown in Table 3 . Thereby $x_{\mathrm{i}}=\dot{V}_{\mathrm{i}} / \dot{V}$ denotes the fraction of the inner volume flow. The temperature of $T=8^{\circ} \mathrm{C}$ is assumed to be constant.

Concerning the heat transfer for foam, fin and wing pipes, we see from the simulations that structure temperatures appreciably larger than fluid temperature occur only at or near the inside of the heated outer wall (see Fig. 6).

The pressure loss calculations above show that the flow regime in the non-circular outer volumes $V_{0}$ is laminar (see values of $R e_{0}$ in Table 3). Classical heat transfer correlations are not valid in this case [18]. Correlations from Shah and London [19] are also not 


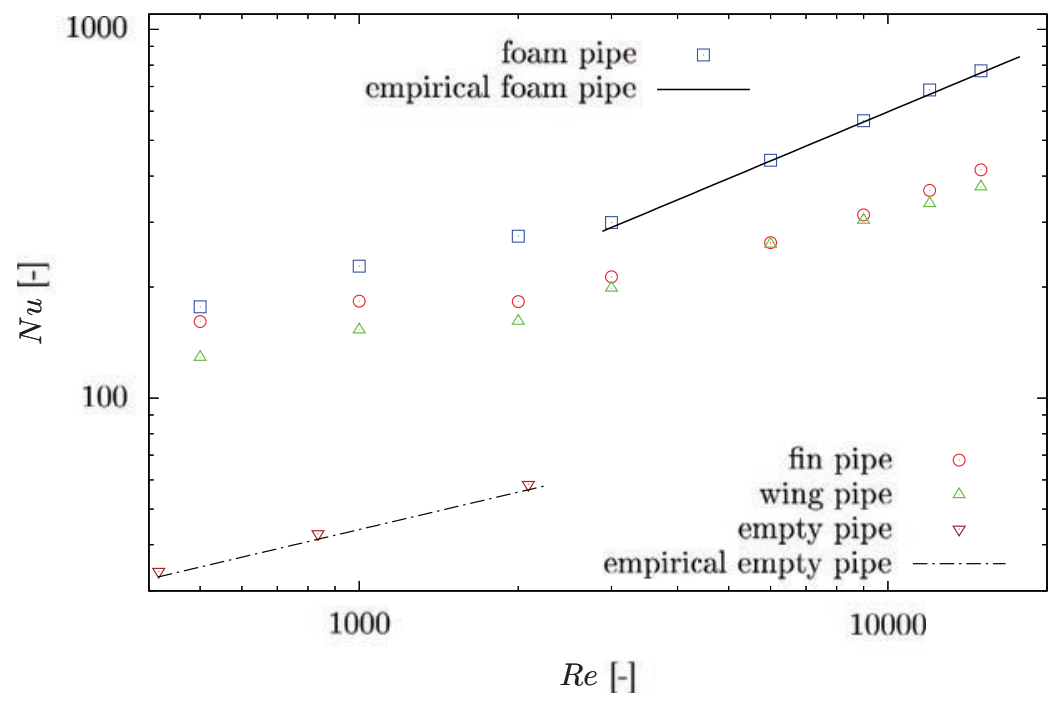

Fig. 8. Nusselt numbers of different pipe structures compared with empty pipe (no inner structure). Symbols show the simulation results and dashed/solid curves refer to the empirical curves for the empty and foam pipes (according to [18], (p. 787, Eq. 19)).

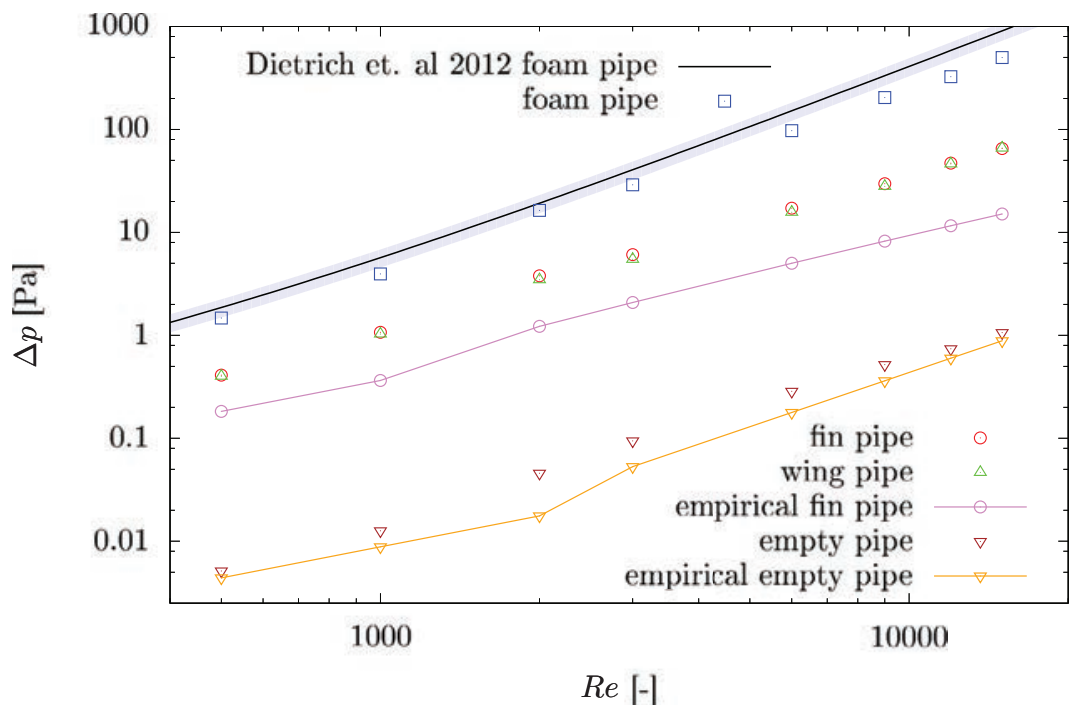

Fig. 9. Pressure drops of different pipe structures compared with an empty pipe (no inner structure). Symbols show the simulation results and lines illustrates the empirical equations.

applicable, as the flow is thermally and hydrodynamically developing in $V_{0}$, so at the device inlet, we locally will have large heat transfer coefficients.

We evaluate the simulation results with regard to the pressure loss and the Nusselt number. Figs. 8 and 9 show the diagrams for the three structures in comparison with each other. In Fig. 9 a correlation for open cell foams presented in [14, Eq.(7)] is also plotted incl. its $20 \%$ error strip. $R e_{\Psi}=\frac{u d_{\mathrm{H}}}{\Psi \nu}$ denotes the porosity Reynolds number and $d_{\mathrm{H}}=4 \frac{\Psi}{S_{V}}$ the hydraulic diameter which is used by Dietrich in [14]. In contrast, in our evaluations of $R e=\frac{u L_{C}}{v}$ we use the pipe diameter as the characteristic length $L_{\mathrm{C}}=D$.

Our results are in good agreement with the experimentally derived correlation given in [14]. Similar to the study of Wakao et al. [20] for packed beds, we can write an empirical correlation for the pipes filled with foams for higher Reynolds numbers as follows:

$N u=1.3 \operatorname{Re}^{0.6} \operatorname{Pr}^{\frac{1}{3}}$.

For further validation, we have performed some simulations with the same heat and mass transfer (laminar) model and with the same input parameters and boundary conditions using the In- stitute's own solver PAcE3D. The results (Fig. 8) show an excellent agreement with the empirical curve calculated using [18], (p. 787, Eq. 19).

Using the ratio of Nusselt to Hagen numbers $(\mathrm{Nu} / \mathrm{Hg})$, the efficiencies concerning the pressure drop of different inner structures can be compared. This is depicted in Fig. 10, where the hydraulic diameters from Table 2 are used to calculate the Hagen number. The fin and wing structures have higher ratios of heat transfer to pressure drop than the foam at all investigated flow rates. Moreover, it clearly illustrates that the ideal operating condition is for the slowest flow. However, if for an application the outlet temperature needs to be maximized, the price to be paid is a higher pressure drop. If this can be accepted, the metal foam would be the better choice.

\section{Experiments}

The pipe elements used in experiments were first designed on computers and partly tested in computer simulations. They were 


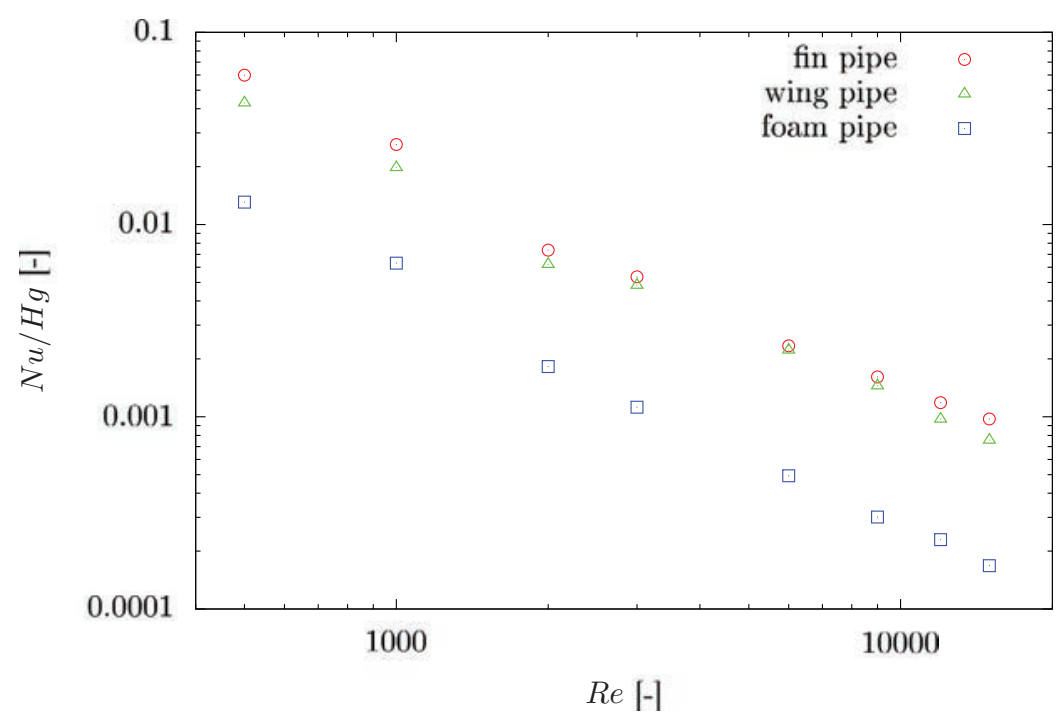

Fig. 10. The ratio of Nusselt to Hagen numbers for different structures pipes.
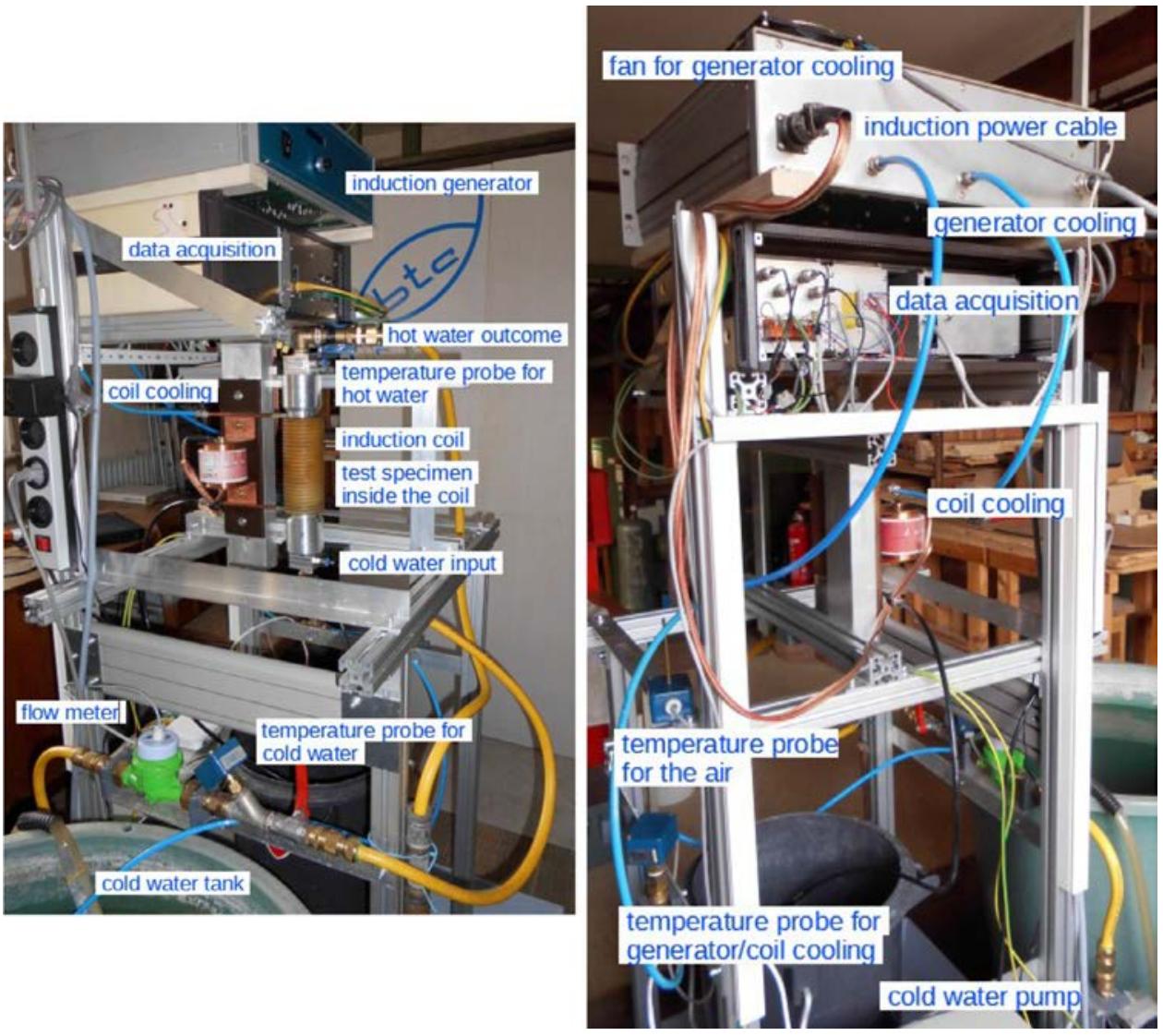

Fig. 11. Left: Induction water heater test rig. Right: Rear side of the test rig.

then 3D-printed using PLA. These 3D prints served as a preform for the investment casting of the actual samples, which were then further processed in the experiment. One tube filled with openpore metal foam was printed using the 3D Selective Laser Melting (SLM) (Fig. 13, (c)). This tube was a particularly high-quality copy of the digital model and was also used to validate the simulations described in Section 3.2.1.

\subsection{Experimental set-up}

The test bench essentially consists of the following components Fig. 11

- Drinking water supply and drainage

- Cold water inlet and hot water outlet 

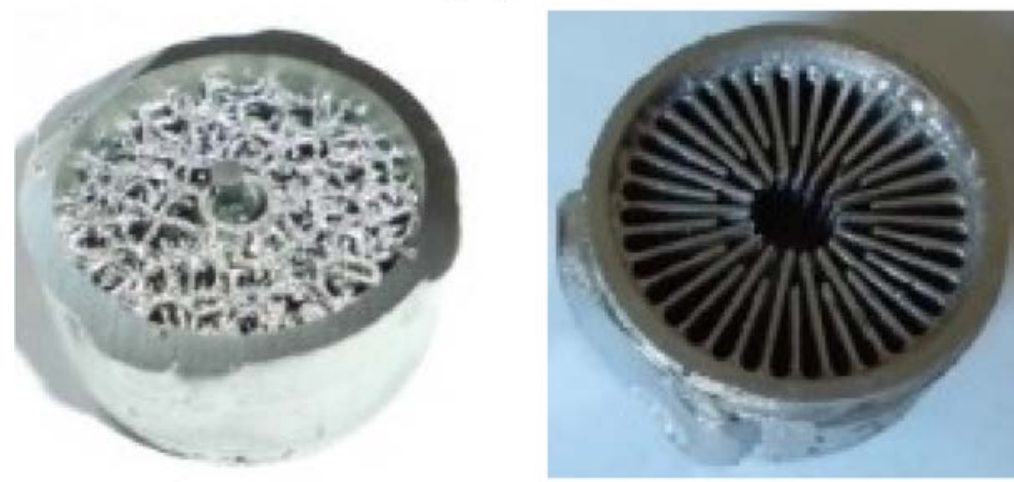

and finish work pieces
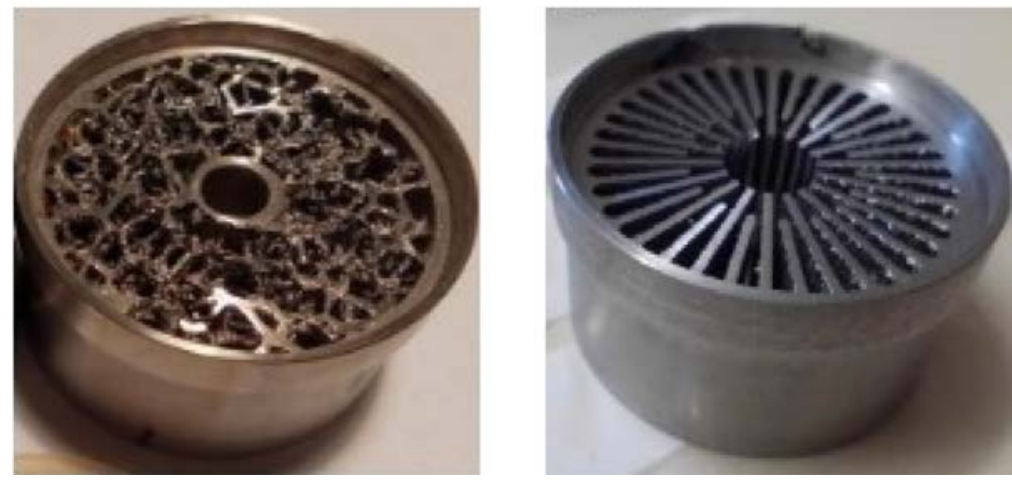

Fig. 12. Metal foam disc and fin disc; centrifugally cast and post-processed (grinding, turning, milling, drilling).

- Induction heating with test specimen holder

- Instrumentation and control engineering with recording and evaluation program as well as logging

The drinking water supply is ensured by a 3-stage adjustable self-priming water pump. The water pump sucks from a water tank. The water is discharged into the receiving water without any retroactive effect via a large hose with a free gradient.

The cooling water supply for the induction coil and for the power semiconductors is ensured by a separate cold water inlet and outlet. Due to the separate water supply, a malfunction of one line is limited to this line. Further damage is avoided.

Induction heating was developed in several stages. It was essential to find a compromise between the partly contradictory requirements from the specifications and the available real heat exchanger material. For the measurement series, a power-controlled induction generator with a maximum output of $6.2 \mathrm{~kW}$ at $400 \mathrm{~V} / 16-20 \mathrm{kHz}$ was finally used. The dimensions of the water-cooled induction coil are based on the realizable centrifugal cast metal foam discs. The MSR technology comprises the sensor technology, the data acquisition and evaluation electronics as well as the transfer of measured values to a PC with logging. Thermocouples are used for measuring structural temperatures, resistance thermometers measure drinking water inlet and outlet temperatures as well as cooling water and ambient temperatures. An impeller flow meter emits one pulse per revolution. All sensors are factory calibrated. The sensor signals are processed by suitable electronic modules that are adapted to two microcontrollers. The microcontrollers have a LAN connection. The PC interface thus corresponds to the current standard. The recording and evaluation program, as well as the logging, runs on a notebook.

\subsection{Test specimen preparation and adapter design}

Two geometrically different metal discs are examined for heat input into the water. As can be seen in Fig. 12, both metal foam and metal fin discs are produced by the investment casting process. In addition, instead of the metal foam discs, a metal foam tube (Fig. 13(c)) is produced by means of 3D laser steel melting the so-called Selective Laser Melting (SLM).

An overview of the composition and assembly of the test tubes is given:

- Nine discs (foam or fins, material numbers 1.4523 and 1.4404 ) with the total length of $126 \mathrm{~mm}$ are sealed against each other to the outside and braced by a central tie rod. The water flows only inside the panes.

- A larger-diameter glass cladding tube accommodates the pane columns of nine foam/ fin discs and ensures water flow and tightness to the outside. The water flows both inside the discs and in the annular gap between the cladding tube and the column (Fig. 13(a)).

- The nine metal foam discs (material number 1.4404) are inserted loosely and offset from each other (unaligned) into the glass cladding tube. The water flow inside the cladding tube is disturbed. Notice that for the descriptive representation only seven metal foam discs are shown in Fig. 13(b).

- A unique steel rod with the same inner foam structure (SLM foam) and the total length of $139 \mathrm{~mm}$ is sealed for water to flow only inside the rod (Fig. 13(c)).

Each experimental setup requires a specific adapter design on the part of the test specimens. The water inlet and outlet, as well 


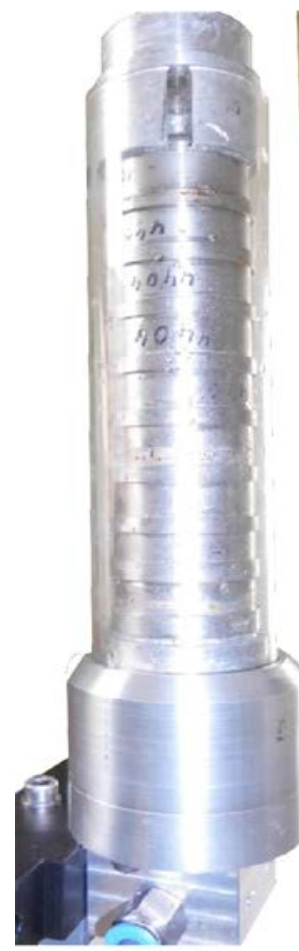

(a)

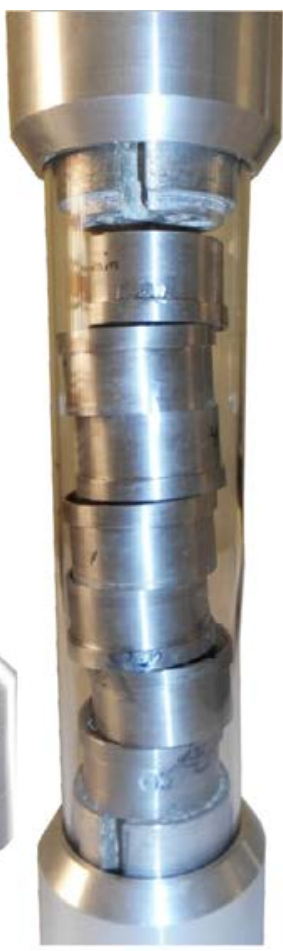

(b)

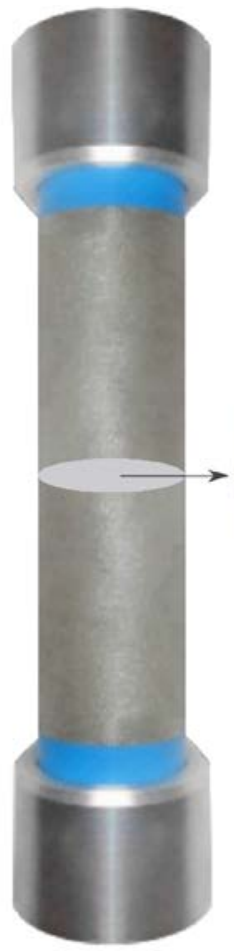

(c)

Fig. 13. (a) and (b) Test tubes, composed of individual discs produced by the investment casting process and (c) test tube produced by the selective laser melting method (SLM).

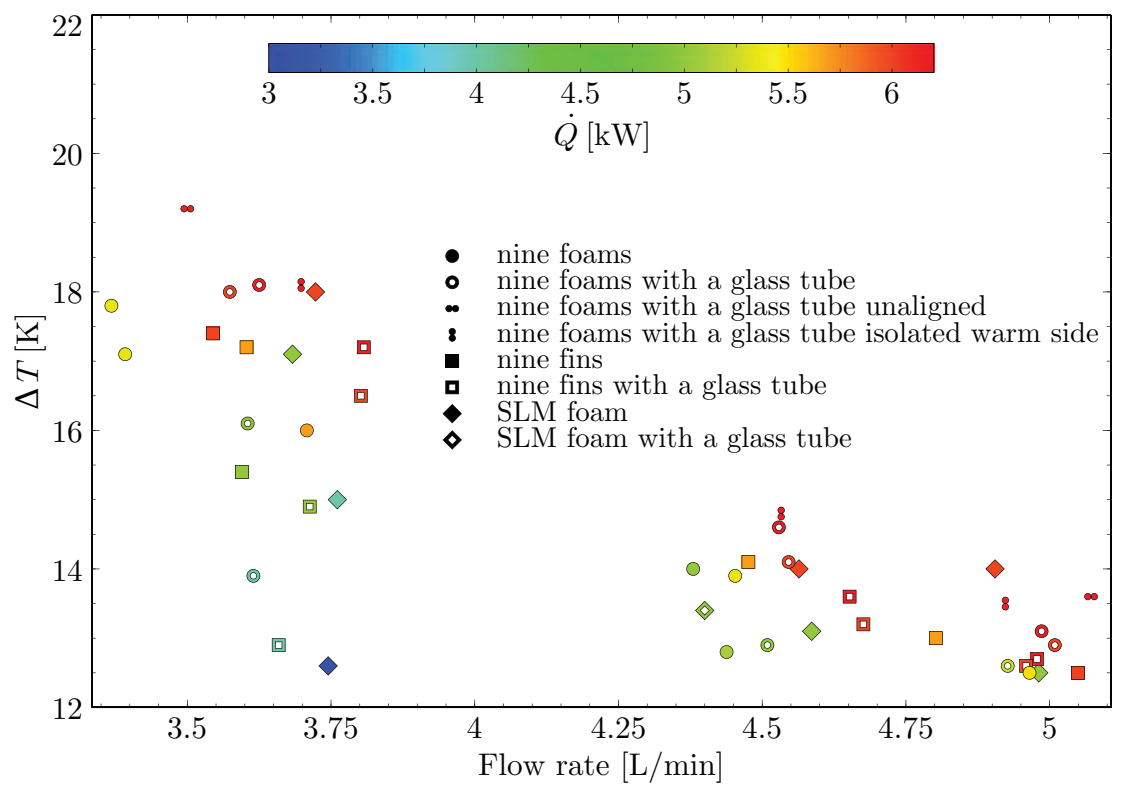

Fig. 14. Temperature difference between inlet and outlet for different flow rates using various experimental setups.

as the thermocouple inlet, remain unchanged. Interchangeable intermediate pieces allow a cost-efficient variety of variants.

The results of the experiments are summarized in Fig. 14.

Higher inductive power causes a stronger temperature rise. Higher flow velocities reduce the temperature rise. Heat transfer is better in foam tubes than in those with fins. The use of glass insulation or a displacement of the individual panes against each other does not bring any significant advantages.

\section{Conclusions}

Surface-rich permeable internal structures of the line pipes increase the heat transfer from the metal to flowing water. In order to classify this phenomenon, we simulatively compared three structures with almost the same porosity and almost the same specific surface. The penetration depth of the heat in the radial direction of the pipe plays an important role in the transfer of 


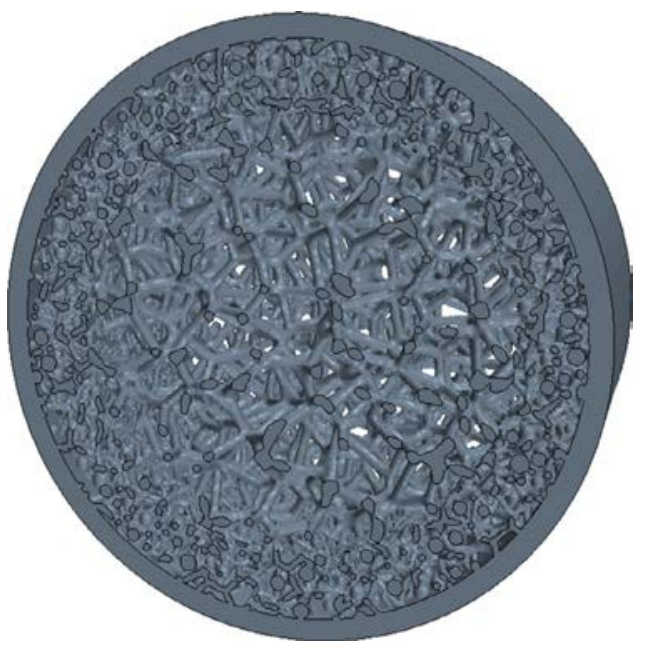

Fig. 15. A pipe lined with open-pored metal foam. The pore structure in the edge area is finer than in the middle.

the heat to the fluid. The greater the contact area of the pipe jacket with the metallic inner workings, the greater the penetration depth. The largest heat transport is detected in the case of metal foam, which leads to a considerably higher Nusselt number compared to fin and wing structures.

The better mixing of the flowing water through the ligaments of the metal foam also promotes heat transfer.

At the same time, the many chaotically arranged metallic obstacles in the metal foam increase the pressure loss and thus increase the energetic operating expenditure. In this sense, the finned and wing tubes perform better than the metal foam tubes. The wing shape does not seem to have any advantage neither for heat transfer nor for pressure drop, being compared to the cuboid fins. The pressure loss is hardly lower, the heat transfer is significantly worse.

\section{Outlook}

Based on the discussed findings, the next challenge is to adjust the geometric parameters of the foam, such as pore size and ligament thickness (and thus the associated parameters such as specific surface area and porosity) in such a way that the heat transfer is as large as possible and the pressure loss is reasonably small.

An idea to master this challenge is to specifically distribute the pore size in the pipe. Smaller pores and thus a larger specific surface at the pipe boundary due to the temperature layer are advantageous for heat transfer. Larger pores close to the middle axis of the pipe allow freer water flow and thus reduce the pressure loss.

An example of such a geometry is shown in Fig. 15. It is a synthetic structure created algorithmically as described in [13].

The structure shown in Fig. 15 consists of the outer shell (cylinder barrel), a fine-pored foam layer close to the outer shell facilitating heat transport and a large-pored foam core along the symmetry axis of the pipe ensuring good flow behaviour with low pressure loss. The structure was composed from two differently porous synthetic foam structures with by means of Boolean operations.

\section{Declaration of Competing Interest}

The authors declare that they have no known competing financial interests or personal relationships that could have appeared to influence the work reported in this paper.

\section{CRediT authorship contribution statement}

Anastasia August: Conceptualization, Methodology, Software, Validation, Formal analysis, Investigation, Data curation, Writing original draft, Writing - review \& editing, Visualization. Farshid Jamshidi: Conceptualization, Methodology, Software, Validation, Formal analysis, Investigation, Data curation, Writing - original draft, Writing - review \& editing, Visualization. Aron Kneer: Conceptualization, Methodology, Software, Validation, Formal analysis, Investigation, Data curation, Writing - original draft, Writing - review \& editing, Visualization. Rainer H. Wolf: Methodology, Data curation, Writing - review \& editing. Michael Wirtz: Methodology, Software, Formal analysis, Writing - review \& editing. Britta Nestler: Writing - review \& editing, Supervision, Project administration, Funding acquisition.

\section{Acknowledgements}

This work was carried out within an AiF cooperation project EmiFoam with the funding reference ZF4102806JA6 within the framework of the program "Central Innovation Program for Small and Medium-Sized Enterprises (ZIM)" on the basis of the resolution of the German Bundestag. We thank the Federal Ministry of Economics and Energy for the financing. We would like to thank our project partners Dr.-Ing. Alexander Matz and Tobias Lott from the Institute for Materials and Materials Technologies (IWWT) at the University of Pforzheim for the production of the stainless steel discs using the investment casting and centrifugal casting processes. Theoretical background and parts of the data analysis have been provided through financial support of the Helmholtz association within the program HGF34 "EMR - Energy Efficiency, Materials and Resources". We would also like to thank Manuela Leoni from the Institute of Production Engineering WBK at the Karlsruhe Institute of Technology for the production of the metal foam pipe using 3D laser steel melting. We thank Leon Geisen for his support in finding good linguistic formulations.

\section{References}

[1] A.M. Matz, B.S. Mocker, D.W. Müller, N. Jost, G. Eggeler, Mesostructural design and manufacturing of open-pore metal foams by investment casting, Adv. Mater. Sci. Eng. (2014)

[2] Q. Zhang, X.-P. Wu, G. Zhao, Y. Li, C. Wang, Y. Liu, X.-Q. Gong, Y. Lu, High-performance PDNI alloy structured in situ on monolithic metal foam for coalbed methane deoxygenation via catalytic combustion, Chem. Commun. 51 (63) (2015) 12613-12616.

[3] S. Guarino, M. Barletta, S. Pezzola, S. Vesco, Manufacturing of steel foams by slip reaction foam sintering (SRFS), Mater. Des. (2012).

[4] T.J. Lu, H.A. Stone, M.F. Ashby, Heat transfer in open-cell metal foams, Acta Mater. 46 (10) (1998) 3619-3635.

[5] A. August, B. Nestler, F. Wendler, M. Selzer, A. Kneer, E. Martens, Efficiency study of metal foams for heat storage and heat exchange, in: Proceedings of the MMM2010 Freiburg Conference Proceedings, 2010, pp. 355-358.

[6] D. Girlich, Solar collector has absorber which is surrounded by housing, where absorber is made of metallic or metallized, netted three-dimensional structure, where three-dimensional structure is open-pored metal foam and closedpored metal foam, 2010,. DE Patent App. DE200,910,022,932.

[7] G.I. Garrido, F.C. Patcas, S. Lang, B. Kraushaar-Czarnetzki, Mass transfer and pressure drop in ceramic foams: a description for different pore sizes and porosities, Chem. Eng. Sci. 63 (21) (2008) 5202-5217.

[8] A. Kopanidis, A. Theodorakakos, E. Gavaises, D. Bouris, 3D Numerical simulation of flow and conjugate heat transfer through a pore scale model of high porosity open cell metal foam, Int. J. Heat Mass Transf. 53 (11) (2010) 2539-2550.

[9] A. Bhattacharya, V.V. Calmidi, R.L. Mahajan, Thermophysical properties of high porosity metal foams, Int. J. Heat Mass Transf. 45 (5) (2002) 1017-1031.

[10] J. Ettrich, Fluid flow and heat transfer in cellular solids, Schriftenreihe des Instituts für Angewandte Materialien, Karlsruher Institut für Technologie ;, KIT Scientific Publishing, Karlsruhe, 2014, doi:10.5445/KSP/1000042041.

[11] J. Ettrich, A. Choudhury, O. Tschukin, E. Schoof, A. August, B. Nestler, Modelling of transient heat conduction with diffuse interface methods, Modell. Simul. Mater. Sci. Eng. 22 (8) (2014) 085006

[12] S. De Schampheleire, P. De Jaeger, K. De Kerpel, B. Ameel, H. Huisseune, M. De Paepe, How to study thermal applications of open-cell metal foam: experiments and computational fluid dynamics, Materials (Basel) 9 (2) (2016) 94. 
[13] A. August, J. Ettrich, M. Rölle, S. Schmid, M. Berghoff, M. Selzer, B. Nestler, Prediction of heat conduction in open-cell foams via the diffuse interface representation of the phase-field method, Int. J. Heat Mass Transf. 84 (2015) 800-808.

[14] B. Dietrich, Pressure drop correlation for ceramic and metal sponges, Chem. Eng. Sci. 74 (2012) 192-199.

[15] A. Kneer, M. Wirtz, S. Yurtsever-Kneer, S. Barbe, A. August, Modern times need enlightened innovation and sophisticated materials, Galvanotechnik 4 (2018).

[16] W. Lu, C.Y. Zhao, S.A. Tassou, Thermal analysis on metal-foam filled heat exchangers. part i: metal-foam filled pipes, Int. J. Heat Mass Transf. 49 (15-16) (2006) 2751-2761.
[17] C.Y. Zhao, W. Lu, S.A. Tassou, Thermal analysis on metal-foam filled heat exchangers. part II: tube heat exchangers, Int. J. Heat Mass Transf. 49 (15-16) (2006) 2762-2770.

[18] V. deutscher Ingenieure, VDI-WÄrmeatlas, VDI-Buch, 11, Springer, Berlin Heidelberg, 2013, doi:10.1007/978-3-642-19981-3.

[19] R.K. Shah, A.L. London, Laminar Flow Forced Convection in Ducts, Academic Press : New York, San Francisco, London, 1978.

[20] N. Wakao, S. Kaguei, T. Funazkri, Effect of fluid dispersion coefficients on particle-to-fluid heat transfer coefficients in packed beds: correlation of Nusselt numbers, Chem. Eng. Sci. 34 (3) (1979) 325-336. 
Karlsruher Institut für Technologie

\section{Repository KITopen}

Dies ist ein Postprint/begutachtetes Manuskript.

Empfohlene Zitierung:

August, A.; Jamshidi, F.; Kneer, A.; Wolf, R. H.; Wirtz, M.; Nestler, B. Development of synthetic open porous structures for improved heat transfer. 2020. International journal of heat and mass transfer, 159. doi: $10.5445 / / R / 1000120675$

Zitierung der Originalveröffentlichung:

August, A.; Jamshidi, F.; Kneer, A.; Wolf, R. H.; Wirtz, M.; Nestler, B. Development of synthetic open porous structures for improved heat transfer. 2020. International journal of heat and mass transfer, 159, Article No.: 120071. doi:10.1016/j.ijheatmasstransfer.2020.120071 